\title{
Patent Urachus with Bladder Prolapse
}

\author{
Thapa $\mathrm{B}^{1}$, Pun $\mathrm{MS}^{2}$
}

\begin{abstract}
We report a case of bladder prolapse through a patent urachus in a term male neonate with a large, red, tubular, mucosa lined mass inferior to the umbilical cord. A cystic mass communicating with fetal urinary bladder was detected in an antenatal ultrasound in a 26 years primigravida at 18 and 26 weeks gestation. The cyst disappeared at 35 weeks and a new solid mass was noted at the fetal abdominal wall. After birth a protruded mucosal mass inferior to the umbilical cord was noted. Urethral catherisation confirmed communication with bladder. On the second day of life excision of urachus, repair, reduction of bladder and reconstruction of abdominal wall was performed. The patient voided well and was discharged on ninth day without any complication.
\end{abstract}

Key words: Urachus, Bladder prolapse

\section{Introduction}

$T^{\mathrm{h}}$ he urachus is an embryological communication between fetal urinary bladder and the umbilicus. The lumen of the urachus is normally obliterated during the fifth or sixth month of embryonic development and becomes the median umbilical ligament which lies in the space of Retzius, between the transversalis fascia anteriorly and the peritoneum posteriorly.There are four main variants: urachal cyst, urachal sinus, urachal diverticulum and patent urachus. Among all patent urachus is rare $(10 \%)$ and is presented as the most severe form ${ }^{1,2}$. This is the first case report of bladder prolapse through a patent urachus in Nepal.

\section{The Case}

A 26 year old primigravida attended an obstetrics antenatal ultrasound screening at 18 weeks of gestation. The mother has no specific history of medication or illness during pregnancy. The ultrasound showed a smooth hypoechoic umbilical cyst with diameter $18 \mathrm{~mm}$ connecting with a bladder via a small duct and the diagnosis: allantoic cyst and patent urachus. Repeated ultrasound at 26 weeks gestation showed no change. The cyst disappeared at 35 weeks and a small solid mass was noted on fetal abdominal wall on Ultrasonography. The kidneys were normal. Bladder could not be assessed properly.
'Dr. Bijay Thapa, MBBS, MS, Registrar, Paediatric Surgeon, ${ }^{2}$ Dr. Madhusudan Pun, MBBS, DCH, MS, Consultant Paediatric Surgeon and HOD. Both from the department of Paediatric Surgery, Kanti Children's Hospital, Kathmandu, Nepal.

\section{Address for correspondence \\ Dr. Bijay Thapa \\ E-mail: bijaytapa@hotmail.com}

\section{How to cite}

Thapa B, Pun MS. Patent Urachus with Bladder Prolapse. J Nepal Paediatr Soc 2014;34(1):68-70.

doi: http://dx.doi.org/10.3126/jnps.v34i1.7877

This work is licensed under a Creative Commons Attribution 3.0 License.

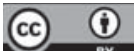

A male newborn of $3000 \mathrm{gm}$ was normally delivered at local hospital in Kathmandu. Patient was immediately referred to Kanti Children's Hospital. The physical examination revealed a $4.5 \mathrm{~cm}$ protruding mass inferior to umbilical cord. Umbilical cord appeared bigger than normal with two arteries and one vein. Other physical examinations were normal. Foleys catherisation per urethra confirmed a communication between the mass and urinary bladder. The exposed mucosa of the bladder had no inflammation or necrosis.

Patient was admitted in SICU and all the necessary investigation done. Patient party was counseled about the case and probable postoperative complications like urinary leakage, wound infections etc. Surgery was performed with incision around the mass along the umbilical ring. The 


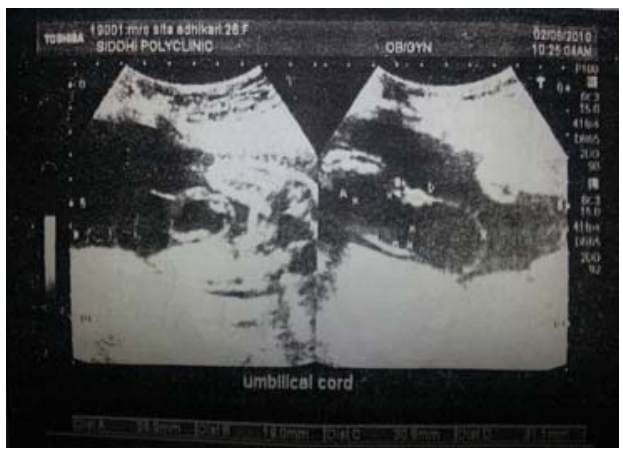

Fig 1: Antenatal USG at 26 weeks

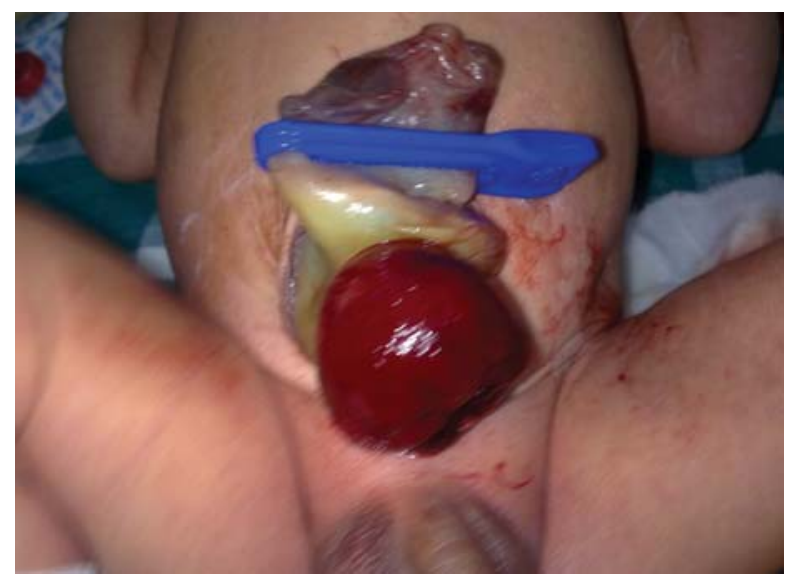

Fig 2: Prolapsed bladder through patent urachus.

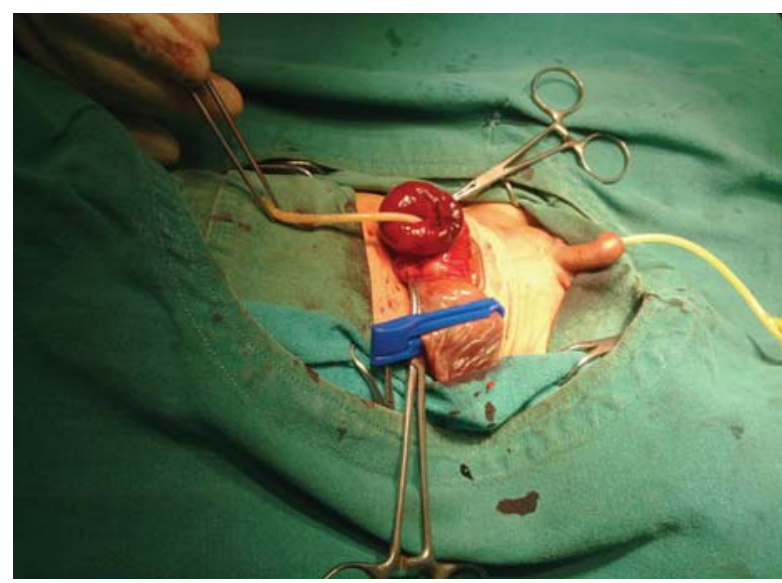

Fig 3: Connection of urethra and bladder

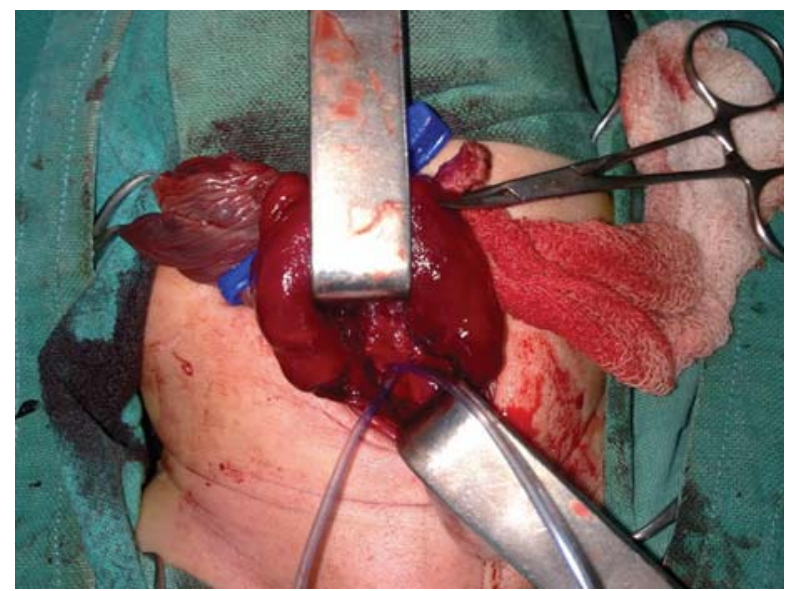

Fig 4: Bilateral ureteric orifices with tubes. umbilical vessels were ligated. The urachus was excised and the bladder was closed in two layers and reduced into the abdomen. The corrugated drain was put and abdominal defect was repaired. The postoperative period was uneventful. Patient started breast feeding next day. Foleys was taken out on sixth day and drain on seventh. Patient voided well and discharged on ninth postoperative day. The first follow-up in OPD showed no complication in two weeks postoperatively.

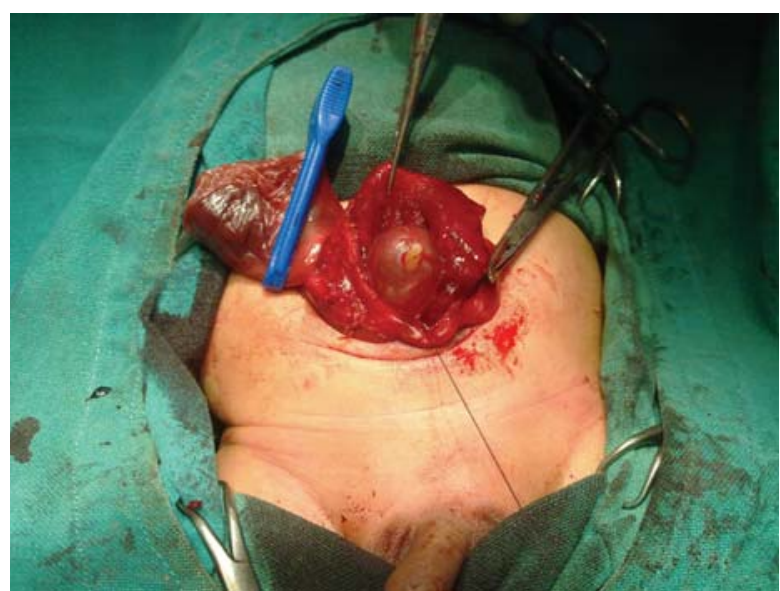

Fig 5: Excision of dome and preparation for bladder closure

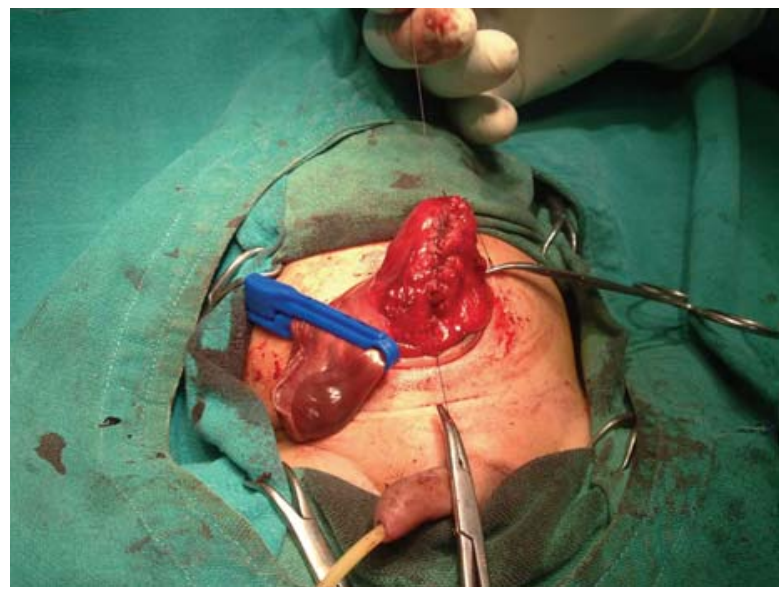

Fig 6: Bladder closure in two layers.

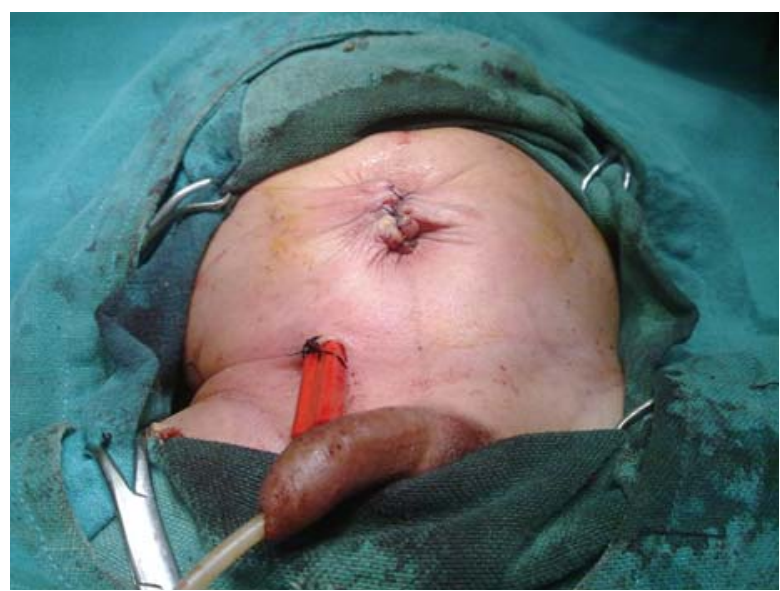

Fig 7: Final appearance of abdominal wall. 


\section{Discussion}

Urachus is an intraembryonic communication between the allantois and the cloaca. It undergoes obliteration of its lumen in the fifth month of gestation while it is stretched by the bladder descending into the pelvis. It narrows to become a fibromuscular strand extending from the apex of bladder to umbilicus which is called median umbilical ligament ${ }^{1}$. The incidence of patent urachus is 0.25:10000 deliveries and males are affected twice as commonly as females².

If this embryological structure persists after birth, it can form one of the four main types. The type of urachal anomaly is directly related to which segment of the urachus persists after birth. Patency at the umbilical end persists as an urachal sinus which may cause umbilical discharge and cellulitis. Patency at the bladder end presents with bladder diverticulum that may be diagnosed later if any clinical symptoms like urinary tract infection or stone formations occurs. Obliteration of both umbilical and bladder end with mid patency results to urchal cyst. Persistence of the whole urachus causes urine leakage through umbilicus ${ }^{2,3}$.

Our case had no associated anomalies as seen on physical examination and abdominal ultrasound. Various associated anomalies have been reported in about $40 \%$ of children with urachal anomalies like omphalomesentric remnants, omphalocele, hydronephrosis, urogenital anomaly etc ${ }^{4}$.

Matsui F et all in their two case reports have stated disappearance of the cyst and appearance of mass in a serial fetal ultrasound which is the characteristic feature of a patent urachus with bladder prolapse. Similar to them our case also has a cystic mass initially which disappeared in late gestation (35th weeks) and changed to a umbilical mass ${ }^{5,6}$.

The cyst may rupture as urine output increases and with the development of the fetal abdominal wall 6,7 . In our case solid mass was observed at 35th weeks of gestation and baby delivered at $37+$ weeks. So due to shorter period of perinatal mucosal exposure there was only minor mucosal inflammation. This may have made bladder closure easier without any postoperative complications.

\section{Conclusion}

In conclusion umbilical anomalies in the neonate include abdominal wall defects, omphalomesentric duct remnants and urachal remnants. Clear diagnosis between these anomalies is necessary because of their possible association with other congenital defects. These different variants may need different workup and different surgical corrections. Early surgical intervention is recommended as early as possible so that prognosis may be better. Patent urachus with bladder prolapse is a rare entity and it is the first case report from Nepal.

\section{References}

1. Lugo B, McNulry J,Emil S. Bladder prolapse through a patent urachus: fetal and neonatal features'. Pediatr Surg 2006;41;5-7.

2. Persutte $W H$, Lenke RR,Kropp K. Antenatal diagnosis of fetal patent urachus. J Ultrasound Med 1988;7:399-403.

3. Jenny H.Yiee, Nilda Garcia, Linda A. Baker. A diagnostic algorithm for urachal anomalies. J Pediatr Urol 2007;3:500-504.

4. Rich R H, Hardy BE, Filler RM. Surgery for anomalies of the urachus. J Pediatr Surg 1983;18:370-2.

5. Yets M,Pinch L.Patent urachus with bladder eversion. J Pediatr Surg 2003;38:12-3.

6. Matsui F,Matsumoto F,Shimada K .Prenatally diagnosed patent urachus with bladder prolapse. J Pediatr Surg 2006;41:e5-7.

7. Hyun Shin Choi,Hae Eun Kim,Eun Sun Kim. Postnatal management of antenatally diagnosed patent urchus with bladder prolapse. J Korean Soc Neonatol 2010;17:262-4. 